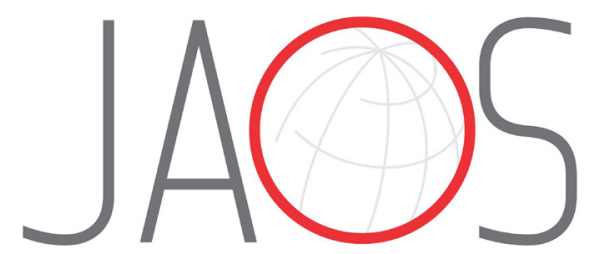

JOURNAL OF APPLIED ORAL SCIENCE

\title{
Assessment of anesthetic properties and pain during needleless jet injection anesthesia: a randomized clinical trial
}

\section{Abstract}

Allan Carlos Araújo de OLIVEIRA ${ }^{1}$

Klinger de Souza AMORIM ${ }^{2}$

Edmundo Marques do

NASCIMENTO JÚNIOR ${ }^{1}$

Amanda Caroline Batista DUARTE ${ }^{1}$

Francisco Carlos GROPPO ${ }^{2}$

Wilton Mitsunari TAKESHITA ${ }^{1}$

Liane Maciel de Almeida SOUZA ${ }^{1}$
Submitted: April 17, 2018 Modification: September 12, 2018

Accepted: September 17, 2018
${ }^{1}$ Universidade Federal de Sergipe, Departamento de Odontologia, Aracaju, Sergipe, Brasil. 'Universidade Estadual de Campinas, Faculdade de Odontologia de Piracicaba, Laboratório de Farmacologia, Anestesiologia e Terapêutica, Piracicaba, São Paulo, Brasil. 


\section{Introduction}

Fear of pain and anxiety in patients is the most notable reason to avoid dental treatment. Injection of local anesthetics is the most painful phase of a treatment ${ }^{1}$ procedure and a significant reason for its premature discontinuation ${ }^{2}$.

There is a relation between anxiety and fear of pain and the actual sensation of pain. Stress induced by anxiety and fear reduces a patient's pain threshold ${ }^{3}$. Moreover, the sensation of pain further results in increased anxiety, and a cycle is established ${ }^{1,2}$.

The efficacy of local anesthetics and the quality standard in needle manufacturing have improved over time. However, the method administrating local anesthetics has practically remained unchanged. Even currently, it is common to use a needle attached to a non-disposable syringe ${ }^{4}$.

Administrating an anesthetic agent with a traditional syringe causes discomfort during the puncture and injection stages ${ }^{5}$. Incorrect handling of the syringe is a determining factor for pain ${ }^{6}$, which is exacerbated due to excessive pressure on the plunger and rapid injection of large volumes of anesthetic solution?

To minimize the painful sensation during local anesthesia, other methods can be adopted, such as applying topical anesthetics prior to injection ${ }^{8}$, using computerized injection systems ${ }^{9}$, manual controlling the injection speed ${ }^{10}$, and using needleless jet injection systems. A needleless system includes a spring coupled to an apparatus that generates sufficient pressure to ${ }^{11}$ push the plunger of the ampoule ${ }^{12}$ and makes the anesthetic solution pass through a microorifice at high speed. According to the manufacturer's recommendations, it administers effective local anesthesia with lower anesthetic volumes compared with the traditional anesthesia method ${ }^{13}$.

The absence of a needle in a jet injection can result in a more comfortable experience, as this eliminates the puncture and injection phases ${ }^{13}$, which are considered the most painful steps during traditional anesthesia ${ }^{5}$. This difference is important as approximately one in five adults have phobia of dental anesthesia due to fear of injections, which leads to interruption of dental treatment ${ }^{14}$.

Since there are few studies about the efficacy of jet injection systems in dentistry, the aim of this the clinical trial was to measure and compare the degree of pain, latency times, and pulpal anesthesia duration in both the traditional method of infiltration anesthesia and needleless jet injection during the treatment of maxillary molars, in a split-mouth trial.

\section{Materials and methods}

\section{Subjects and ethical considerations}

A randomized, single-blind, split-mouth clinical trial was performed. Experimental design followed the Consolidated Standards of Reporting Trials (CONSORT) guidelines; the experimental flow chart is shown in Figure 1. Our local ethics committee approved the study under the protocol CAAE 62481316.4.0000.5546, and it was registered on the Brazilian Registry of Clinical Trials (RBR-9V37H9). The clinical trial took place from January to November 2017.

Sample-based calculation indicated a requirement for 41 volunteers for an $80 \%$ chance of detecting a 10-mm difference in the degree of pain measured by the visual analogue scale (VAS) at a significance level of $5 \%{ }^{15,16}$. Participants were volunteers who were being treated at the Department of Dentistry of the Federal University of Sergipe, of both genders, with ages between 18 and 40 years, and who required dental restorations in the maxillary first molars with middepth class I carious lesions and had healthy maxillary second molars reactive to electric stimulation.

The exclusion criteria were evaluated based on medical history and clinical examination. We excluded individuals with history of allergy or other problems related to any of the components of the anesthetic solution, those with fear of dental treatment, alcoholics and drug users, individuals using analgesics or medications acting on the central nervous system, pregnant women, and individuals undergoing treatment with appliances and orthodontic bands.

All patients signed an informed consent form prior to dental treatment.

\section{Anesthetic methods}

The needleless jet injection method used was the Comfort-in system (Mika Medical; Busan, Korea) and the traditional infiltration anesthesia method was a carpule syringe with a 30-gauge short needle. Lidocaine 2\% with epinephrine 1:100,000 (DFL Ind. Com. SA; Rio de Janeiro, Rio de Janeiro, Brazil) was used as anesthetic and the volume was standardized to $1.0 \mathrm{ml}$ for both methods. The anesthetic methods were 
performed only by an operator that had experience in carrying out both the jet anesthesia technique and oral surgery. For traditional anesthesia, $0.8 \mathrm{ml}$ of the solution of each anesthetic tube was withdrawn with a standard Comfort-in adapter. The carpule syringe (Duflex, S.S. White; Rio de Janeiro, Rio de Janeiro, Brazil) was coupled with a 30-gauge stainless steel short gingival needle (Becton Dickinson; São Paulo, São Paulo, Brazil). The needle was inserted in the muco-vestibular fold above the apex of the maxillary second molars ${ }^{6}$.

Topical anesthetics were not used to avoid interference with the perception of pain ${ }^{16}$. As a result, the total time for anesthetic injection was set at 1.5 minutes because a slow injection decreases the chances of tissue rupture on contact with the anesthetic solution. Consequently, there may have been a reduction in the discomfort experienced during injection ${ }^{6}$.

In the jet method, the anesthetic solution was administered in a fractional manner. Four ampoules were used: the first was filled with $0.1 \mathrm{ml}$, and the other three were filled $0.3 \mathrm{ml}$ of the anesthetic, as recommended by the manufacturer ${ }^{13}$.

The equipment had a pressurized spring and a silicone cap (recto cap) coupled with the ampoule containing the anesthetic solution for preserving the periodontal tissues. The jet injection system was positioned at $90^{\circ}$ in relation to the maxilla with slight compression next to the gingival band inserted at the second maxillary molar. The inserted gingiva was delimited by the mucogingival junction and the coronal (free) gingiva ${ }^{17}$ of the maxillary second molar.

Anesthesia was administered by pressing a button to release the anesthetic solution. The volunteers were informed about the noise produced by the equipment during the release of the anesthetic solution to prevent reflex reactions.

\section{Outcomes}

At the end of each anesthetic method, the pain sensation due to injection was measured using VAS. The volunteer was required to make a vertical line on the $100 \mathrm{~mm}$ line, indicating the pain level experienced during anesthesia. A digital caliper measured these values later.

The second maxillary molars were electrically stimulated by the Micro-controlled Digital Pulp Tester (Microeletrônica Indústria e Comércio Ltda-ME; Belo Horizonte, Minas Gerais, Brazil) in the first session to determine the threshold value of the basal electrical stimulation before administrating the anesthetic solution. Only one operator manipulated this device to ensure a standardized protocol.

The pulp electric tester (PET) electrode was positioned on the middle third of the vestibular face of the tooth. At first, the equipment was used with minimum amperage (0 $\mathrm{mA})$, which was gradually increased until the individual reported sensitivity. At this point, the basal threshold value was defined. The

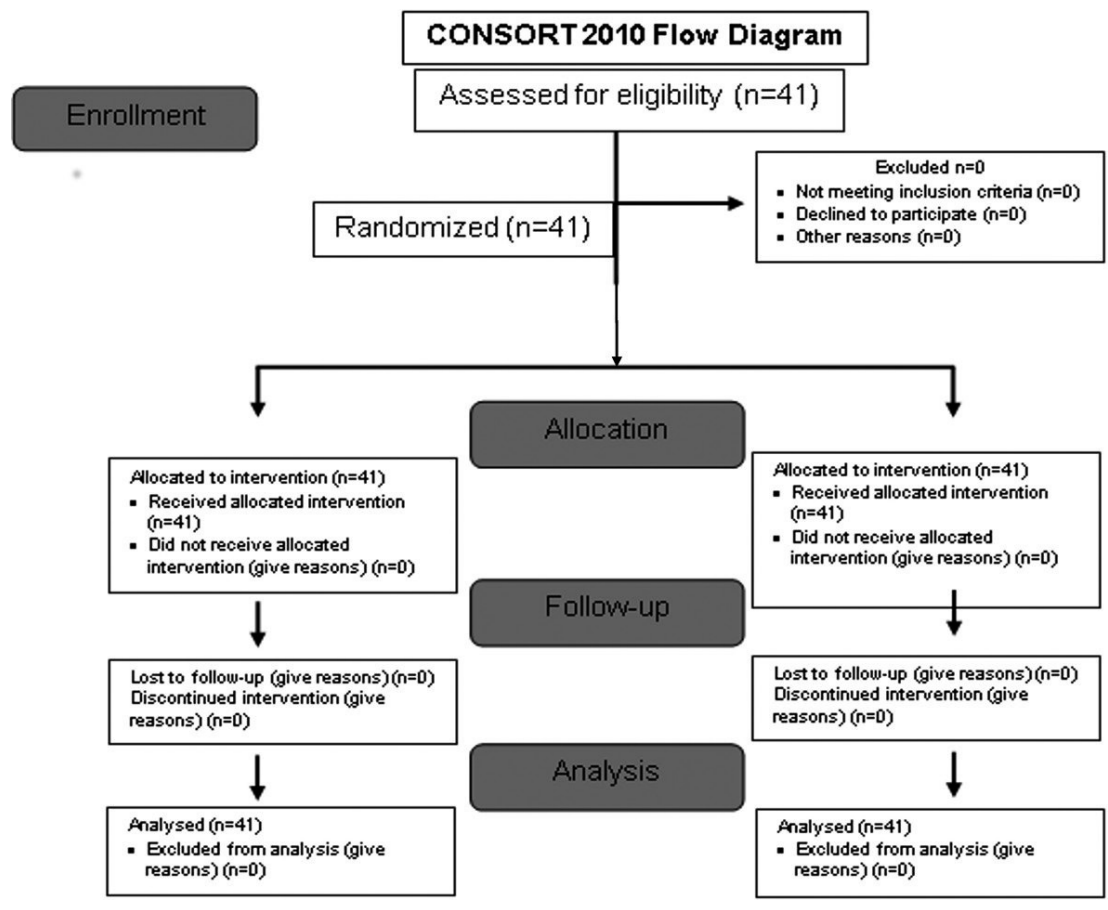

Figure 1- CONSORT flow diagram of patients enrolled in the clinical trial 
maximum amperage used in the study was $80 \mathrm{~mA}^{18}$.

Anesthetic latency time: this was defined as the period immediately after injecting the anesthetic to the onset of anesthetic effect. The absence of sensitivity to the electrical stimulus in two cycles of $80 \mathrm{~mA}$ confirmed the beginning of the effect and pulpal anesthetic efficacy and defined the latency time measured in 2 and 5 minutes. Anesthesia failure was considered when the volunteer showed sensitivity to electrical stimulus at the tenth minute after anesthetic injection ${ }^{19}$.

Duration of pulpal anesthesia: this corresponded to the period between the beginning of anesthetic action and the moment the tooth returned to its basal electrical stimulation threshold. The maxillary second molars were tested every 10 minutes with the application of $80 \mathrm{~mA}$ stimuli until they returned to the baseline threshold value ${ }^{19}$

\section{Clinical study protocol}

The randomization list was created by the evaluator from the website www.sealedenvelope.com. Randomization was applied to the anesthetic method and the laterality of the maxilla. The split-mouth design resulted in all volunteers undergoing anesthesia using both anesthetic methods (needleless jet injection and traditional infiltration anesthesia). To ensure a blind study, information regarding the randomization was enclosed in an opaque envelope and known only to the operator.

The complete clinical trial protocol required three sessions. After defining the basal electrical stimulation threshold, the volunteer received local anesthesia using either the needleless jet injection or traditional infiltration anesthesia method performed by the operator. The interval between sessions was set at 7 days, and the effect of drug metabolism on pain threshold was excluded ${ }^{20}$.

At the time of anesthesia, the clinician responsible for measuring parameters was not present at the outpatient clinic in accordance with the single-blind study design. In the second minute after anesthetic injection, the PET measured latency time. If the tooth still responded to electrical stimulus, it was stimulated again in the fifth minute after anesthesia.

Glass ionomer cement (Maxxion R ${ }^{\circledR}$ FGM; Joinville, Santa Catarina, Brazil) was used for temporary restorative treatment of the maxillary first molars. It was inserted and accommodated into the clean cavity according to the manufacturer's recommendations, and the process was finished with occlusal adjustment.

The restorative procedure did not exceed 10 minutes. Every 10 minutes, new electrical tests were performed on the maxillary second molar until it returned to the basal electrical stimulation threshold. In this way, the duration of pulpal anesthesia was measured.

In the third clinical session, the volunteers received definitive restorative treatment with an adhesive system (Ambar Universal FGM; Joinville, Santa Catarina, Brazil) and composite resin (Filtek-Z350, 3M-ESPE, São Paulo, São Paulo, Brazil). No adverse effect was observed during the anesthetic procedures.

\section{Statistical analysis}

The numerical variables were analyzed using the Shapiro-Wilk test to verify normality and the Bartlet test to determine the homoscedasticity of their variances The percentage analysis was applied to distribute the genders and choose the anesthetic technique. The t-test was used to analyze the differences between the groups for the basal electrical stimulation threshold values. The duration of anesthesia and degree of pain measured using VAS were compared using the Mann-Whitney test. A 5\% significance level was considered for all tests, and they were performed using the BioEstat 5.0 statistical packages (Instituto Mamirauá, Belém, Pará, Brazil) and GraphPad Prism 7.0 (GraphPad software, La Jolla, California, United States).

\section{Results}

Of the 41 volunteers, 23 (56.1\%) were male and $18(43.9 \%)$ female, and there was no statistical difference $\left(x^{2}, p=0.53\right)$ between their proportion. The mean age was $25.7( \pm 4.4)$ years, which also showed no significant difference (unpaired t-test, $p=0.43$ ). According to the t-test, the basal electrical stimulation threshold did not show any statistical difference between its values measured before administration of anesthesia using the two anesthetic methods $(p=0.188)$ (Figure 2).

The Mann-Whitney test showed no statistical difference in the VAS pain during anesthesia $(p=0.571)$ between the two methods. The VAS was 12.2 (0 $55.4)$ for the needleless jet method and 12.1 ( 0 - 53.8) for the traditional infiltration anesthesia (Figure 3 ). 


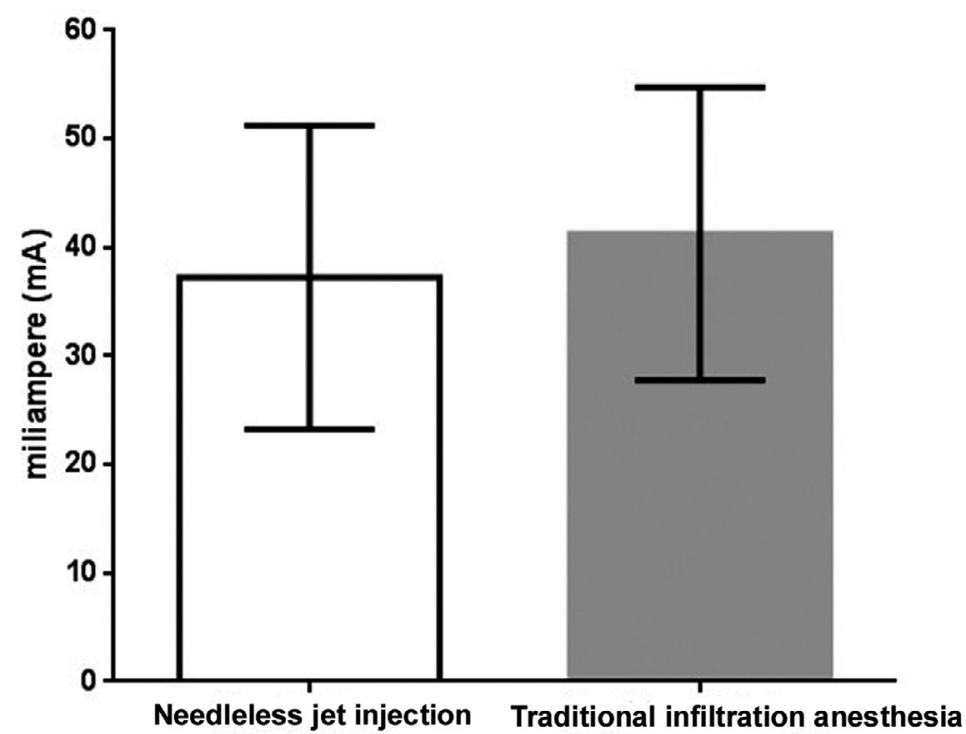

Figure 2- Basal electrical stimulation threshold (mean \pm standard deviation) as a function of anesthetic method. Student's $T$ test, $p>0.05$

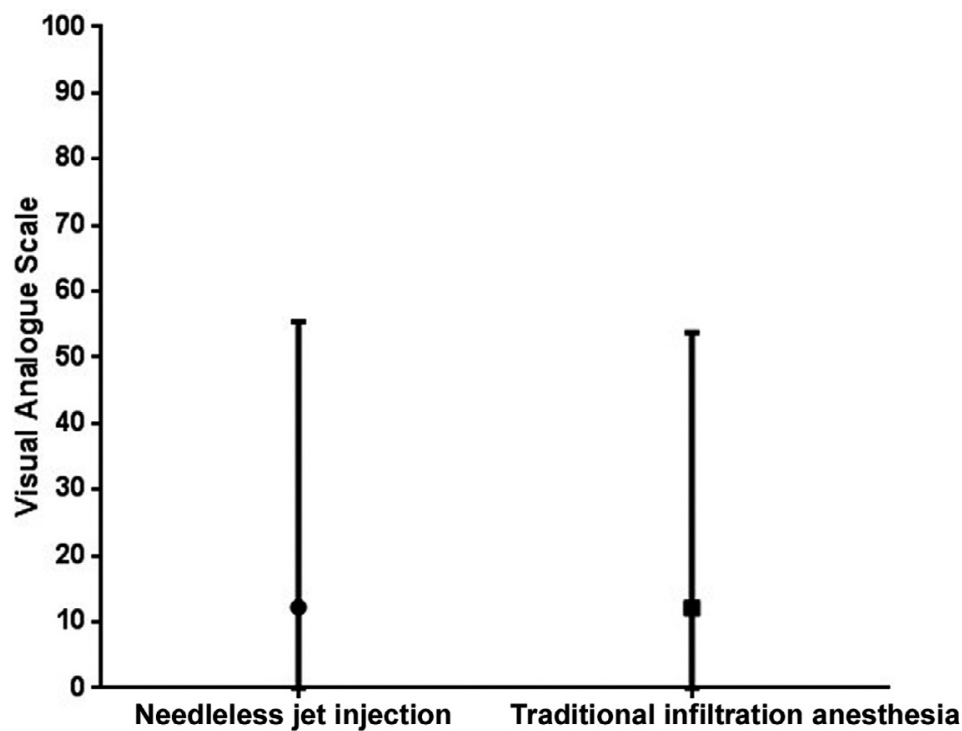

Figure 3- VAS as a function of the degree of pain experienced during administration of anesthesia using the two anesthetic methods in the maxilla. Center bar represents median, maximum, and minimum values. Mann-Whitney test, $p>0.05$

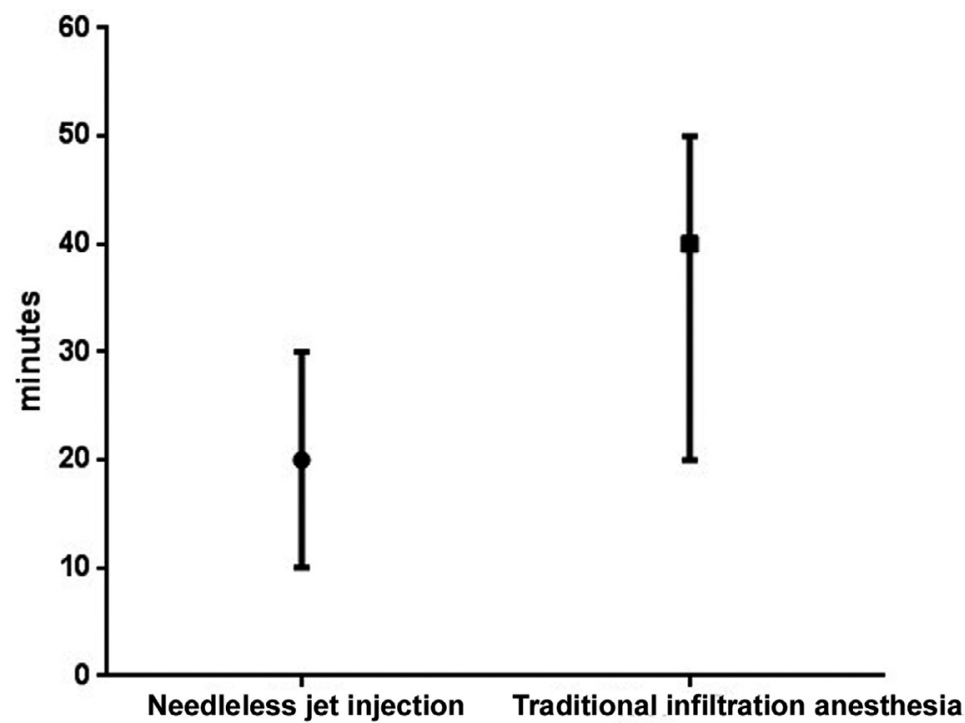

Figure 4- Pulpal anesthesia duration (minutes) for the two anesthetic methods. Center bar represents median, maximum, and minimum values. Mann-Whitney test, $p<0.001$ 
The latency time recorded for the two anesthetic methods was 2 minutes for all subjects.

The Mann-Whitney test showed a significant difference in pulpal anesthesia duration (minutes) between the two methods $(p<0.001)$. The median duration of pulpal anesthesia for the needleless jet injection and traditional infiltration anesthesia was 20.0 and 40.0 minutes, respectively (Figure 4 ).

No volunteers required additional anesthesia at any stage of the restorative procedure.

\section{Discussion}

The need for local dental anesthesia should be determined according to the clinical situation and should be administered with minimal pain sensation ${ }^{21}$. Pain during dental anesthesia has a negative impact on the patient. In this study, for both anesthetic methods, the median values were within the score range considered to indicate low degree of pain. This is contrary to the results of a study that compared pain levels during anesthesia between the WAND electronic system and the Injex needleless jet injection system; the mean pain was higher for the needleless jet injection system ${ }^{22}$.

Anesthetic latency and duration of anesthesia are important parameters for planning clinical procedures under local anesthesia. In this study, the determined anesthetic latency time was 2 minutes for both anesthetic methods. This parameter defines the onset speed of anesthetic action and can vary according to the anesthetic agent as well as modifications in anesthetic techniques ${ }^{23}$. Lidocaine has a low dissociation constant (7.7 pKa), resulting in a low anesthetic latency time ${ }^{6,24}$ of 2 to 4 minutes $^{6}$. In this study, the aim was not to specifically test the drug's latency, but rather to evaluate anesthetic latency relative to the method of administration, using the needleless jet injection.

Another important finding in this study was that the duration of pulpal anesthesia was lower for the jet injection compared with the traditional syringe injection. This may be attributed to pharmacokinetic processes that take place during tissue diffusion after injecting the anesthetic. The greater is the initial concentration of local anesthetic in the nonionized form at the injection site, the faster is the diffusion, with unimpeded movement of these liposoluble molecules towards the nerve fascicles in the epineurium ${ }^{6}$.

The local anesthetic diffusion is not unidirectional. A jet injection that deposits the entire anesthetic volume in a fraction of a second possibly allows a higher rate of diffusion by providing a high anesthetic concentration at one time. When it diffuses into the nerve, the local anesthetic becomes progressively more diluted because of extracellular tissue fluids. At this point, nonneural tissues, the capillaries and lymphatic vessels in the region of infiltration, also absorb the drug. As the concentration of extraneural local anesthetic decreases, the concentration of local anesthetic within the nerve rises with progressing diffusion until the concentrations are balanced, and then they begin to reverse. The nerve impulses relaying pain to the brain remain blocked only until the local anesthetic is present in the nerve, and this time period defines the duration of anesthesia ${ }^{6}$.

The possible increase in the speed of all these events due to the high initial concentration of the anesthetic solution after the jet injection compared with the slow infiltration of the anesthetic with a traditional syringe can explain the significant difference in pulpal anesthesia durations.

One clinical trial determined that the mean pulpal anesthetic duration for the traditional technique is $50 \pm 9.32$ minutes $(p>0.001)$ with a short needle and carpule syringe, whereas the jet injection technique resulted in the lowest duration of $20 \pm 3.53$ minutes $(p>0.001)^{25}$. These results are consistent with the findings of our study.

The needleless jet injection eliminates the puncture and needle insertion phases, which may make injection of the anesthetic less painful. However, the pulpal anesthesia duration reported in our study can be considered insufficient for dental procedures such as endodontic treatment and dental extraction. Minor periodontal clinical procedures and class I dental restorations can be satisfactorily performed under pressure anesthesia, provided the treatment is completed within 20 minutes. However, this depends on the skill and experience of the clinician performing the procedure.

The absence of the use of a method to assess the degree of anxiety in individuals prior to anesthesia and the impossibility of a design in which the volunteer remains blind during the administration of anesthesia may be considered as limitations of our 
study. Moreover, future studies should be conducted to evaluate anesthetic duration with different volumes of lidocaine, other anesthetic drugs, and by applying pressure anesthesia not only the maxilla, but also other to areas of the mandible.

\section{Conclusions}

The two anesthetics methods did not differ concerning pain experienced during the anesthesia. The anesthetic latency was 2 minutes for all subjects, and the traditional infiltration anesthesia resulted in a longer anesthetic duration when compared with the needleless jet injection.

\section{References}

1- Lalabonova CK. Impact of dental anxiety on the decision to have implant treatment. Folia Med (Plovdiv). 2015;57(2):116-21.

2- Raghav K, Van Wijk A, Abdullah F, Islam MN, Bernatchez M, De Jongh A. Efficacy of virtual reality exposure therapy for treatment of dental phobia: a randomized control trial. BMC Oral Health. 2016;16:25. 3- Glaesmer $H$, Geupel $H$, Haak R. A controlled trial on the effect of hypnosis on dental anxiety in tooth removal patients. Patient Educ Couns. 2015;98(9):1112-5.

4- Dabarakis NN, Alexander V, Tsirlis AT, Parissis NA, Nikolaos M. Needle-less local anesthesia: clinical evaluation of the effectiveness of the jet anesthesia Injex in local anesthesia in dentistry. Quintessence Int. 2007;38(10):E572-6.

5- Kuzin AV. Practical advices in choosing local anesthesia tools in dentistry. Management of carpule's quality in local anesthesia in dentistry. Stomatologiia (Mosk). 2014;93(2):37-9.

6- Malamed SF. Manual de anestesia local. 6th ed. Rio de Janeiro, RJ: Elsevier; 2013.

7- Rood JP. The pressures created by inferior alveolar injections. $\mathrm{Br}$ Dent J. 1978;144(9):280-2.

8- Al-Melh MA, Andersson L. Comparison of topical anesthetics (EMLA/Oraqix vs. benzocaine) on pain experienced during palatal needle injection. Oral Surg Oral Med Oral Pathol Oral Radiol Endod. 2007;103(5):e16-20.

9- Friedman MJ, Hochman MN. The AMSA injection: a new concept for local anesthesia of maxillary teeth using a computer-controlled injection system. Quintessence Int. 1998;29(5):297-303.

10- Kanaa MD, Meechan JG, Corbett IP, Whitworth JM. Speed of injection influences efficacy of inferior alveolar nerve blocks: a double-blind randomized controlled trial in volunteers. J Endod. 2006;32(10):919-23.
11- Munshi AK, Hegde A, Bashir N. Clinical evaluation of the efficacy of anesthesia and patient preference using the needle-less jet syringe in pediatric dental practice. J Clin Pediatr Dent. 2001;25(2):131-6. 12- Gozdemir M, Demircioglu RI, Karabayirli S, Sert H, Muslu B, Usta $B$, et al. A Needle-Free Injection System (INJEXTM) with lidocaine for epidural needle insertion: a randomized controlled trial. Pak J Med Sci. 2016;32(3):756-61.

13- Instruction Manual. Comfort-intm Needle Free Injection System [Internet]. [cited Jan 22 2017]. Available from: http://www.comfort-in. com/file/English-French\%20manual.pdf.

14- Kleinknecht RA, Thorndike RM, McGlynn FD, Harkavy J. Factor analysis of the dental fear survey with cross-validation. J Am Dent Assoc. $1984 ; 108(1): 59-61$.

15- Lwanga SK, Lemeshow S. Sample size determination in health studies: a practical manual. Geneva: World Health Organization; 1991. 16- Meechan JG, Day PF. A comparison of intraoral injection discomfort produced by plain and epinephrine-containing lidocaine local anesthetic solutions: a randomized, double-blind, split-mouth, volunteer investigation. Anesth Prog. 2002;49(2):44-8.

17- Lindhe J, Karring T, Lang NP. Tratado de periodontia clínica e implantologia oral. 5th ed. Rio de Janeiro: Guanabara Koogan; 2010. 18- Vähätalo $K$, Antila $H$, Lehtinen R. Articaine and lidocaine for maxillary infiltration anesthesia. Anesth Prog. 1993;40(4):114-6.

19- Silva CB, Groppo FC, Santos CP, Serpe L, Franz-Montan M, Paula $E d$, et al. Anaesthetic efficacy of unilamellar and multilamellar liposomal formulations of articaine in inflamed and uninflamed tissue. $\mathrm{Br}$ J Oral Maxillofac Surg. 2016;54(3):295-300.

20- Bender IB, Landau MA, Fonsecca S, Trowbridge HO. The optimum placement-site of the electrode in electric pulp testing of the 12 anterior teeth. J Am Dent Assoc. 1989;118(3):305-10.

21- Kämmerer PW, Schiegnitz E, von Haussen T, Shabazfar N, Kämmerer $\mathrm{P}$, Willershausen $\mathrm{B}$, et al. Clinical efficacy of a computerised device (STATM) and a pressure syringe (VarioJect INTRATM) for intraligamentary anaesthesia. Eur J Dent Educ. 2015;19(1):16-22.

22- Zarzecka J, Gończowski K, Kesek B, Darczuk D, Zapała J. Comparison of the systems used for providing local anesthesia in dentistry--the Wand (Milestone Scientific) and Injex (Rosch). Przegl Lek. 2006;63(12):1304-9.

23- Britto AC, Oliveira AC, Lima CA, Souza LM, Paixão MS, Groppo $F C$, et al. Comparison of the anesthetic latency of articaine, lidocaine, levobupivacaine and ropivacaine, through "Pulp Tester." Rev Odontol UNESP. 2014 Jan;43(1):8-14

24- Strichartz GR, Sanchez V, Arthur GR, Chafetz R, Martin D. Fundamental properties of local anesthetics. II. Measured octanol:buffer partition coefficients and pKa values of clinically used drugs. Anesth Analg. 1990;71(2):158-70

25- Makade C, Shenoi P, Gunwal M. Comparison of acceptance, preference and efficacy between pressure anesthesia and classical needle infiltration anesthesia for dental restorative procedures in adult patients. J Conserv Dent. 2014;17(2):169-74. 